\title{
Evaluación de una planta piloto para el tratamiento de aguas residuales ordinarias por medio de un filtro percolador con relleno de esponjas colgantes de flujo descendente (DHS) como postratamiento de un efluente de sedimentador primario
}

\author{
Assessement of a pilot plant for the treatment of domestic wastewater \\ with a reactor of Downflow Hanging Sponge (DHS) for the posttreatment \\ of a primary sedimentation effluent
}

\author{
Erick Centeno Mora \\ Universidad de Costa Rica, Escuela de Ingeniería Civil \\ MSc. Gestión y tratamiento del agua, el suelo y los desechos e Ingeniero Civil. \\ erick.centenomora@ucr.ac.cr \\ Diego Rodríguez Cambronero \\ Ingeniero Civil \\ dirocam@hotmail.com \\ Jose Luis Ugalde Herra \\ Dirección de Recolección y Tratamiento de la Subgerencia de Gestión de Sistemas de la GAM \\ Instituto Nacional de Acueductos y Alcantarillados, Ingeniero Civil \\ jugalde@aya.go.cr
}

Recibido: 13 de setiembre 2017

Aceptado: 17 de noviembre 2017

\section{Resumen}

La investigación consistió en evaluar un filtro percolador con material de relleno de espuma de poliuretano para el postratamiento de un efluente de sedimentador primario. A esta tecnología se le conoce como DHS por sus siglas en inglés para Downflow Hanging Sponge. El tren de tratamiento se completó con un sedimentador secundario (SS) a la salida del DHS. El sistema DHS + SS se monitoreó por más de un año (entrada al DHS y salida del SS), haciendo variar el tiempo de retención hidráulica y revisando las eficiencias de remoción de materia orgánica en términos de la DBO, DQO y los SST.

El sistema presentó su mejor desempeño para un tiempo de retención hidráulica de 2,8 horas, equivalente a una carga orgánica volumétrica de entre 0,60 a $0,80 \mathrm{kgDBO} / \mathrm{m}^{3}-\mathrm{d}$. Para esa condición se cumplió con los límites de DBO y DQO del DE-33601-S-MINAE en el $100 \%$ de los casos, aunque se incumplió en un $25 \%$ del tiempo para el parámetro de SST. Se presume que el sistema presentó problemas a nivel del sedimentador secundario, generando un arrastre de lodos que afectó el resultado de SST en el efluente final.

Se recomienda ampliar la escala de la prueba con el reactor DHS, usando una planta de tratamiento de escala real y mejorando sustancialmente el sistema de distribución de agua a la entrada y el sedimentador secundario. 
Palabras clave:

Tratamiento de aguas residuales, planta piloto, filtro percolador, esponjas colgantes de flujo descendente, DHS, postratamiento de efluente primario.

\begin{abstract}
This research assessed the operation of a trickling filter filled with sponge media of polyurethane for the posttreatment of a primary clarifier effluent. This technology is known as Downflow Hanging Sponge (DHS). The treatment process was completed by a secondary clarifier (SS). The system DHS + SS was monitored for more than a year (inflow of the DHS and outflow of the SS), varying the hydraulic retention time (HRT) and following its performance for the organic matter removal.

The system had his best behavior for a HRT of 2,8 hours, for an organic volumetric loading rate between 0,60 to $0,80 \mathrm{kgDBO} / \mathrm{m}^{3}-\mathrm{d}$. For that condition, the system achieved the limits of BOD and COD established in the Costa Rican regulation in $100 \%$ of time, although the local limit for the parameter of the TSS was not reached in $25 \%$ of time. It is presumed that the system had some troubles at the level of the secondary clarifier, producing a sludge washing-out that jeopardized the final effluent quality.

It is recommended to extend the scale of the DHS system, using a real wastewater treatment plant and upgrading the design for the distribution system at the inlet and for the secondary clarifier.
\end{abstract}

Keywords:

Wastewater treatment, pilot plant, trickling filter, downflow hanging sponges, DHS, posttreatment of primary sedimentation. 


\section{INTRODUCCIÓN}

La búsqueda de tecnologías sostenibles para el tratamiento de las aguas residuales ordinarias en Costa Rica es uno de los objetivos más importantes de la Política Nacional de Saneamiento en Aguas Residuales (AYA, MINAE \& MINSA, 2016).

Se espera que en los próximos 30 años el desarrollo en infraestructura de alcantarillado sanitario y sistemas de tratamiento de aguas residuales se acelere significativamente para mejorar la situación del país en esta materia, que ya en el año 2014 provocó que Costa Rica descendiera del puesto 3 al 54 en el Índice de Desempeño Ambiental de la Universidad de Yale, cuando ese mismo año se agregó el indicador de porcentaje de tratamiento de aguas residuales a este índice (AYA et al., 2016).

Una de las decisiones más importantes a la hora de resolver el tema del tratamiento de las aguas residuales es la escogencia del proceso de tratamiento (Metcalf \& Eddy, 2014). Existen muchos trenes de tratamiento y, particularmente para el tratamiento secundario o biológico, muchas tecnologías pueden aplicarse para la remoción de la materia orgánica, principal objetivo del tratamiento de las aguas residuales en Costa Rica, según lo establecido en el Reglamento de Vertido y Reuso de Aguas Residuales (DE-33601-S-Minae, 2007).

En Costa Rica, se estima que más del 75\% de los sistemas de tratamiento tramitados en APC (la plataforma del Colegio Federado de Ingenieros y Arquitectos por la que cualquier proyecto constructivo debe ser aprobado) corresponde a la tecnología de lodos activados de aireación extendida (Murillo, 2017), ocupando las otras tecnologías menos del $25 \%$ de los proyectos de tratamiento de aguas residuales tramitados (incluyendo otras modalidades de los lodos activados). Aunque la tecnología de lodos activados ha probado ser eficiente y adecuada para el tratamiento de las aguas residuales ordinarias desde hace más de 100 años (Metcalf \& Eddy, 2014), suele ser una tecnología de elevado costo operativo debido a la alta demanda energética y a la elevada producción de lodos. Estos costos deben ser transferidos a la población servida, lo que produce una tarifa elevada para el servicio de tratamiento de las aguas residuales. En los casos en los que la población servida no es capaz de sostener los costos operativos, la planta suele ser abandonada, con el impacto ambiental correspondiente sobre el cuerpo de agua que recibe las aguas residuales sin ningún tratamiento.

Con el fin de explorar otras opciones tecnológicas para el tratamiento biológico de las aguas residuales ordinarias aplicables para el contexto de Costa Rica, se evaluó un filtro percolador con medio de soporte formado por espuma de poliuretano con sedimentador secundario, como postratamiento de un sedimentador primario. A este tipo de filtro percolador se le conoce también como DHS por siglas en inglés para Down-flow Hanging Sponge (Harada, 2015) traducido al español como esponjas colgantes de flujo descendente. Se trata de una tecnología desarrollada inicialmente en Japón, la cual se ha usado principalmente para el postratamiento de reactores anaerobios de flujo ascendente de manto de lodos (UASB por sus siglas en inglés), aunque existen algunas pocas referencias sobre su uso para postratamiento de procesos de remoción de sólidos por sedimentación primaria a escala de laboratorio (Sh Uemura, Suzuki, Maruyama, \& Harada, 2012).

El sistema DHS + SS se probó variando la carga hidráulica de operación del sistema y monitoreando la calidad del agua de entrada al reactor y salida del sedimentador 
secundario por más de un año, a escala de planta piloto. Se monitorearon tres parámetros de calidad representativos de la remoción de la materia orgánica y de seguimiento obligatorio, según la reglamentación nacional correspondiente (DE-33601-S-Minae, 2007): Demanda Biológica de Oxígeno (DBO), Demanda Química de Oxígeno (DQO) y Sólidos Suspendidos Totales (SST).

\section{MATERIALES Y MÉTODOS}

La planta piloto de DHS + SS se construyó en la Planta de Tratamiento de Aguas Residuales (PTAR) Los Tajos de AyA, usando el efluente final del sistema de tratamiento existente. El proceso de tratamiento de la PTAR Los Tajos para la línea de agua consiste en un desbaste, desarenado y sedimentación primaria.

Para la construcción de la planta piloto se siguió el esquema experimental mostrado en la siguiente figura.

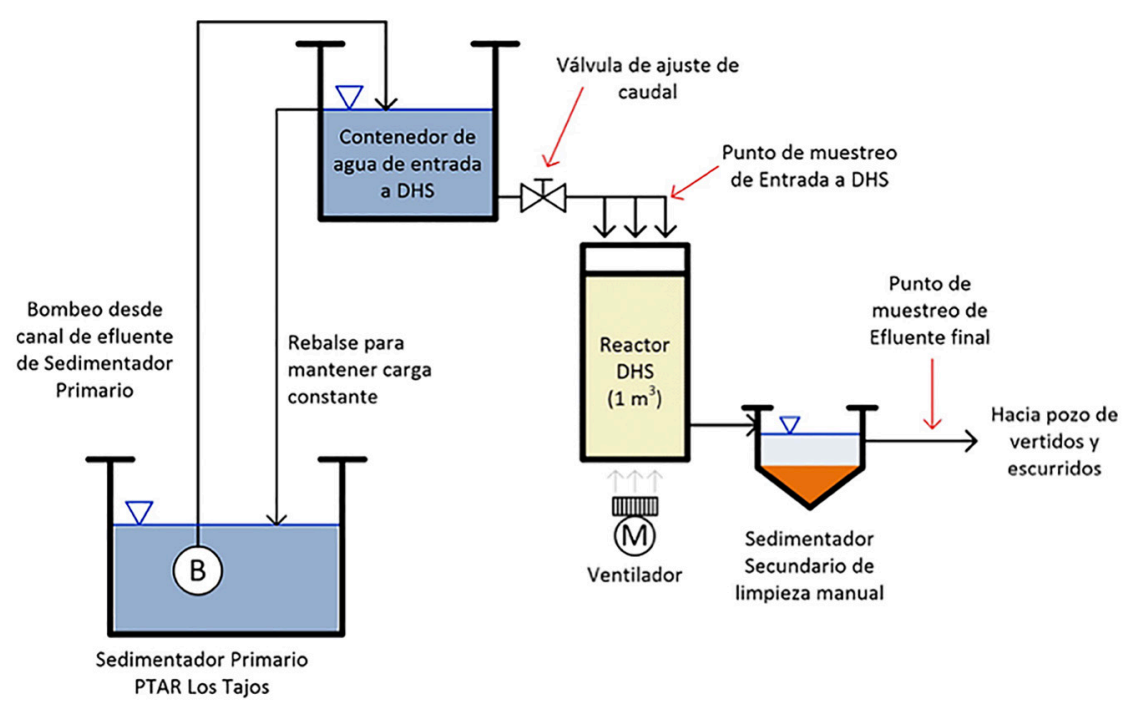

Figura 1. Montaje experimental de planta piloto.

En el canal de salida del tratamiento primario de este sistema se colocó una bomba para extraer el agua cruda necesaria para el reactor piloto de DHS. Por lo tanto, se trabajó con un efluente de tratamiento primario, en el cual se esperaban concentraciones relativamente bajas de sólidos suspendidos totales y una contaminación orgánica (DBO, DQO) principalmente soluble y no sedimentable.

Mediante el bombeo se envió el agua residual efluente del tratamiento primario hacia un tanque de agua con un nivel constante para garantizar una carga hidráulica constante a lo largo de los distintos caudales evaluados. Posteriormente este tanque fue eliminado y el control de caudal se dio mediante manejo directo de válvulas. Se ajustaba el caudal a evaluar por medio de una válvula y el agua era repartida sobre el medio de soporte usando 
canaletas. El reactor tenía un volumen útil de espuma de $1 \mathrm{~m} 3$. En el fondo se instaló un ventilador con una capacidad de hasta $85 \mathrm{~m} 3 / \mathrm{h}$ de caudal de aire. El efluente del reactor DHS era enviado a un sedimentador secundario de limpieza manual de aproximadamente $0,25 \mathrm{~m} 2$ de área superficial con tiempo de retención hidráulico de entre 10 a 25min.

En la siguiente figura se muestra una fotografía de algunos elementos del sistema.

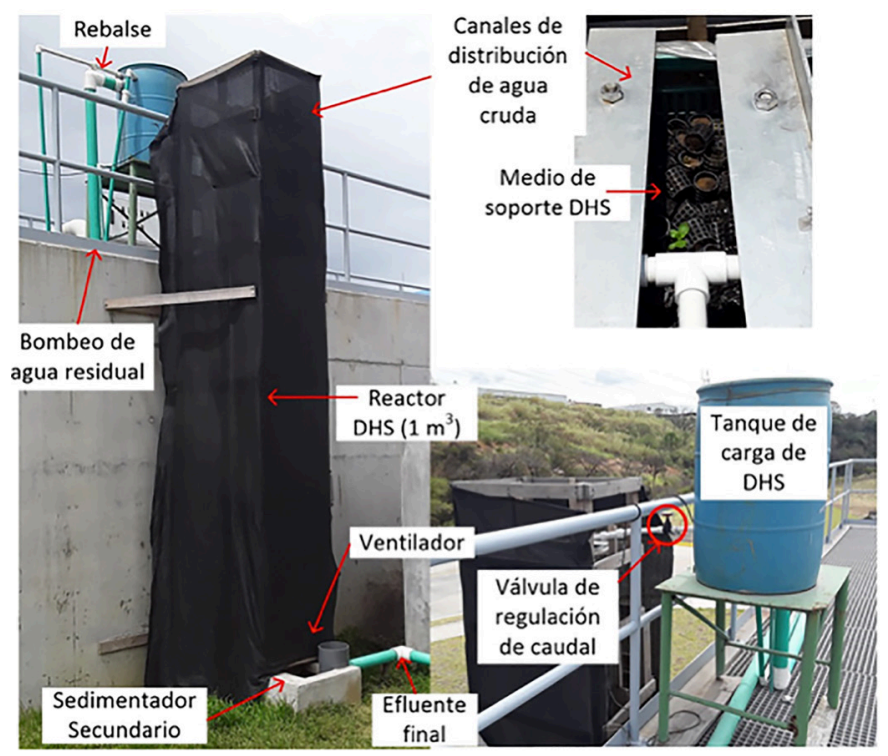

Figura 2. Fotografía de Planta piloto de DHS + SS y sus elementos.

La altura del reactor DHS era de aproximadamente 4,5m y se colocó el medio de soporte en cajas plásticas canaladas, como se muestra en la siguiente figura. Se usaron 12 cajas de dimensiones de $37 \mathrm{~cm}$ de alto, $70 \mathrm{~cm}$ de largo y $40 \mathrm{~cm}$ de ancho.

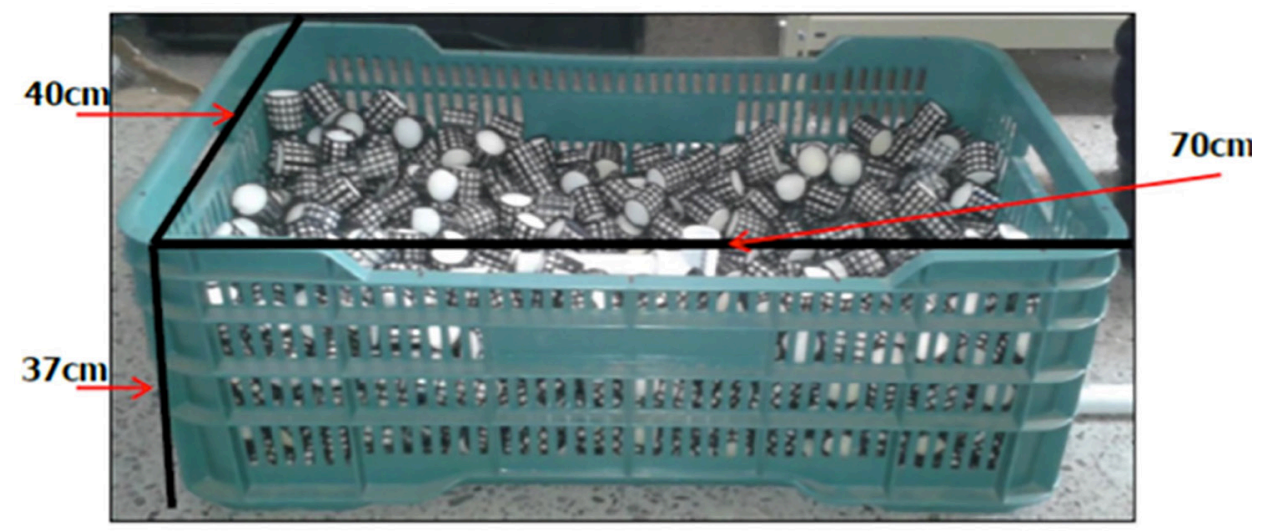

Figura 3. Cajas de material de soporte DHS (previo a arranque de planta piloto) Fuente: Rodríguez, 2017. 
El material de relleno fue donado por la empresa japonesa NJS y la Universidad Tecnológica de Nagaoka en Japón. Se utilizó espuma de poliuretano en forma de cilindros con dimensiones aproximadas de $2,5 \mathrm{~cm}$ de diámetro por $3 \mathrm{~cm}$ de alto, como se observa en la siguiente figura. La espuma está dentro de un soporte de plástico que se utiliza para que la espuma no se deforme durante el proceso de tratamiento y al mismo tiempo se conserve el espacio vacío entre cada elemento de soporte. El material utilizado corresponde al DHS de tercera generación (Harada, 2015).

Para el seguimiento operacional se consideraron los parámetros de DBO, DQO y SST. En la siguiente tabla se muestran los procedimientos seguidos durante la fase de análisis de laboratorio. Se consideraron muestras compuestas de dos horas, tanto a la entrada del DHS como a la salida del Sedimentador Secundario de limpieza manual. En la medida de lo posible, se tomó al menos un muestreo del agua de entrada y de salida por semana. Para los análisis de laboratorio se utilizó el laboratorio Ambiental de la Escuela de Ingeniería Civil de la Universidad de Costa Rica. Adicionalmente se contó con dos muestreos y análisis de laboratorio realizados por el Laboratorio Nacional de Aguas AyA, los cuales incluyeron análisis compuestos de dos horas al afluente y efluente del sistema DHS + SS.

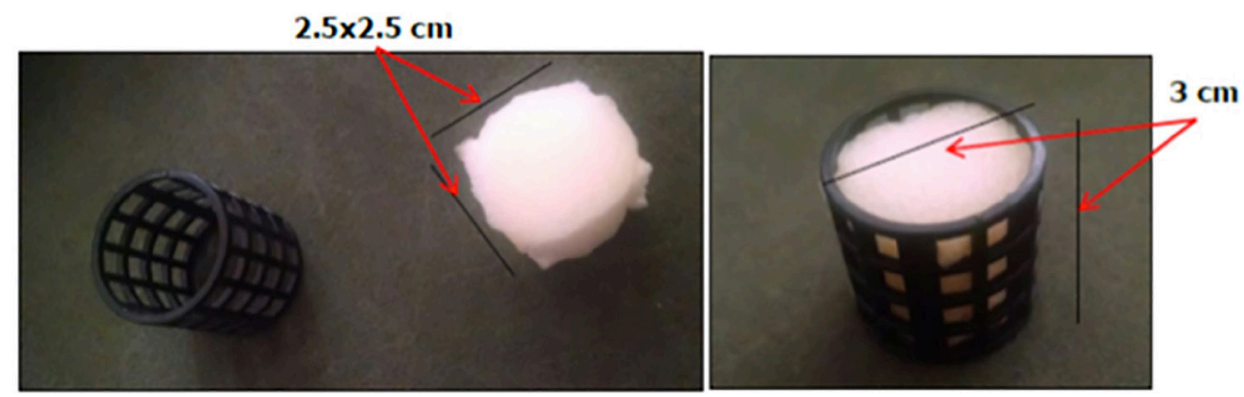

Figura 4. Elemento de espuma de poliuretano. Fuente: Rodríguez, 2017

El período de evaluación inició desde mediados de marzo del 2016 hasta aproximadamente mayo del 2017. Durante este período se consideraron las siguientes condiciones:

- Período de puesta en marcha: se trabajó a un caudal de 3 1/min (Tiempo de Retención Hidráulica, TRH, en el reactor de 5,5h y Tasa de Aplicación Hidráulica Superficial, TAH de $15 \mathrm{~m} 3 / \mathrm{m} 2-d)$. Cuando se consideró que la biomasa se había formado se aumentó el caudal de trabajo.

- Período de análisis estacionario: se trabajó a un caudal de 6 1/min (TRH en el reactor de $2,7 \mathrm{~h}$ y TAH de $31 \mathrm{~m} 3 / \mathrm{m} 2$-d ) durante un período de varios meses y se observó el comportamiento del reactor a nivel de calidad del efluente y otras condiciones de operación del sistema. Al final de esta etapa se detuvo momentáneamente el seguimiento del reactor y se retomó posteriormente, estabilizándolo nuevamente en 6 1/min.

- Período de falla del sistema: se aumentó la carga hidráulica para provocar la falla en el sistema. El valor de carga hidráulica que produjo la falla fue a partir de $10 \mathrm{l} /$ min (TRH en el reactor de $1,7 \mathrm{~h}$ y TAH de $51 \mathrm{~m} 3 / \mathrm{m} 2-\mathrm{d}$ ). 
El TRH se calcula en función del volumen de espuma de poliuretano $\left(1 \mathrm{~m}^{3}\right.$ en este caso). Este parámetro suele usarse para la evaluación de los sistemas DHS (Harada, 2015; Tawfik, Ohashi, \& Harada, 2006; S. Uemura et al., 2016; Sh Uemura et al., 2012) debido a que el funcionamiento del sistema parte del hecho de que el agua saturará la esponja y realmente se almacenará en el volumen de esponja disponible para la depuración. La TAH es un parámetro usual para la evaluación del funcionamiento de los filtros percoladores (Bressani Ribeiro, 2015; Metcalf \& Eddy, 2014; Von Sperling, 2007).

Para todas las etapas se realizaron análisis de laboratorio, con excepción de la última etapa de falla, debido a problemas en los análisis de laboratorio ajenos al control del experimento. Aun así se presentan resultados cualitativos para esta etapa.

Tabla 1 Parámetros de seguimiento operacional y métodos de medición

\begin{tabular}{|l|c|c|c|}
\hline Parámetro & Unidades & $\begin{array}{c}\text { Método de medición de } \\
\text { Standard Methods }\end{array}$ & Equipo de medición \\
\hline Caudal, Q & $1 / \mathrm{min}$ & Volumétrico & NA \\
\hline $\begin{array}{l}\text { Demanda Biológica de } \\
\text { Oxígeno, DBO* }\end{array}$ & $\mathrm{mg} / 1$ & 5210D respirométrico & Hach Bod Trak I \\
\hline $\begin{array}{l}\text { Demanda Química de } \\
\text { Oxígeno, DQO }\end{array}$ & $\mathrm{mg} / 1$ & 5220D & $\begin{array}{c}\text { Hach DR900, } \\
\text { Method 8000 }\end{array}$ \\
\hline $\begin{array}{l}\text { Sólidos Suspendidos } \\
\text { Totales, SST }\end{array}$ & $\mathrm{mg} / 1$ & 2540D & $\begin{array}{c}\text { Horno marca } \\
\text { Equatherm y filtro } \\
\text { marca BOECO con } \\
\text { tamaño de poro entre } \\
12 \text { y 15 } \mu \mathrm{m}\end{array}$ \\
\hline
\end{tabular}

* Se corrigió el valor obtenido de DBO por respirometría para hacer corresponder el valor obtenido con el del procedimiento 5210B de Standard Methods, más usado en Costa Rica (Rodríguez, 2017).

\section{RESULTADOS}

\subsection{Parámetros de calidad del agua}

A continuación se presentan los resultados de los análisis de agua a la entrada del reactor DHS y salida del sedimentador secundario. Se muestran los resultados experimentales para los parámetros de DBO, DQO y SST.

La eficiencia de remoción de los distintos contaminantes fue calculada como:

$$
E=\frac{C_{e}-C_{s}}{C_{e}} \cdot 100 \%
$$

Donde

E: eficiencia de remoción del contaminante especificado (100\%) 
Ce: concentración de entrada al DHS del contaminante especificado (mg/l)

Cs: concentración de salida del sedimentador secundario del contaminante especificado $(\mathrm{mg} / \mathrm{l})$

En los gráficos mostrados a continuación se pueden observar también las fases del experimento: puesta en marcha, estado estacionario, pausa en el seguimiento y período de recuperación del sistema. En el primero de ellos se observa la evolución de la DBO en el tiempo.

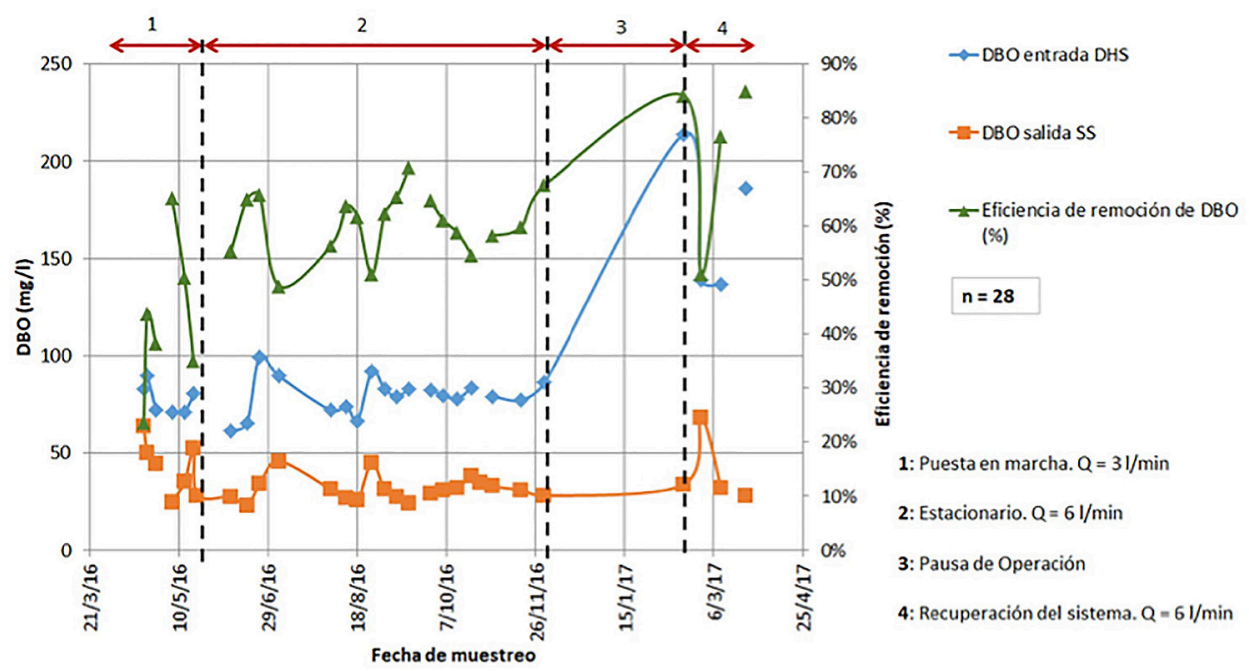

Figura 5. Variación de la DBO en el sistema de DHS +SS durante el período de evaluación

En el caso de la DBO, la tendencia de la calidad del agua en el efluente sigue un comportamiento similar al de la calidad del agua de entrada, manteniendo una eficiencia de remoción general de entre 50 a $70 \%$ de la DBO. Además se observa que los valores del agua de entrada del DHS corresponden a valores típicos de calidad de efluentes en sedimentadores primarios para aguas residuales ordinarias (Metcalf \& Eddy, 2014)

Para el período 2, en donde se tenían condiciones casi estacionarias (un sistema estable a nivel de TRH), se cumplió en todos los muestreos con el límite de vertido de DBO de 50 mg/l. Únicamente no se cumplió con este límite durante la puesta en marcha del sistema y durante el inicio de la etapa de recuperación del reactor.

En la siguiente figura se presenta el comportamiento de la DQO durante el período de evaluación.

Con respecto a la DQO, el agua tratada cumple para todos los muestreos excepto uno, durante la puesta en marcha. En el período 2 de carga casi estacionaria, cuando el sistema se estabilizó, se cumplió con el 100\% de los muestreos y el sistema se recuperó rápidamente una vez que se echó a andar durante la fase 4 de recuperación. En el caso de la eficiencia de remoción de DQO en el reactor DHS, ese parámetro varió entre $41 \%$ a $80 \%$, rango de valores usuales para el sistema biológico (Metcalf \& Eddy, 2014). 


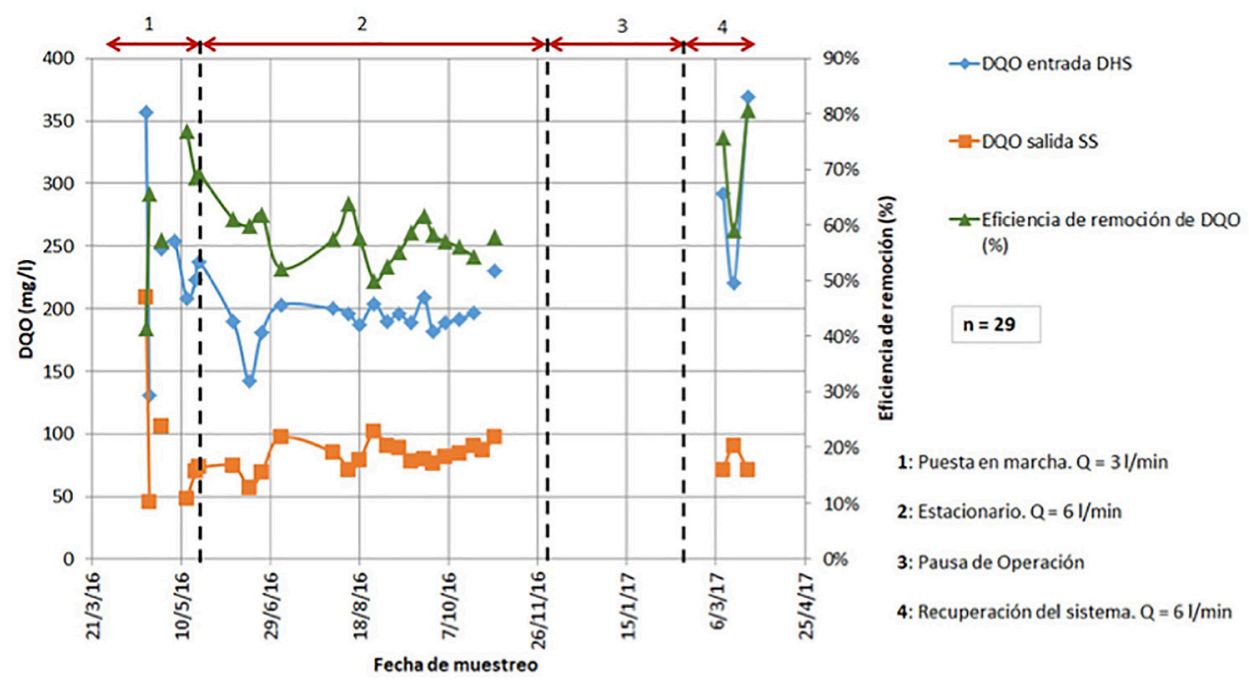

Figura 6. Variación de la DQO en el sistema de DHS durante el período de evaluación.

En la siguiente figura se muestra el comportamiento del parámetro de SST en el sistema de DHS durante el período de evaluación.

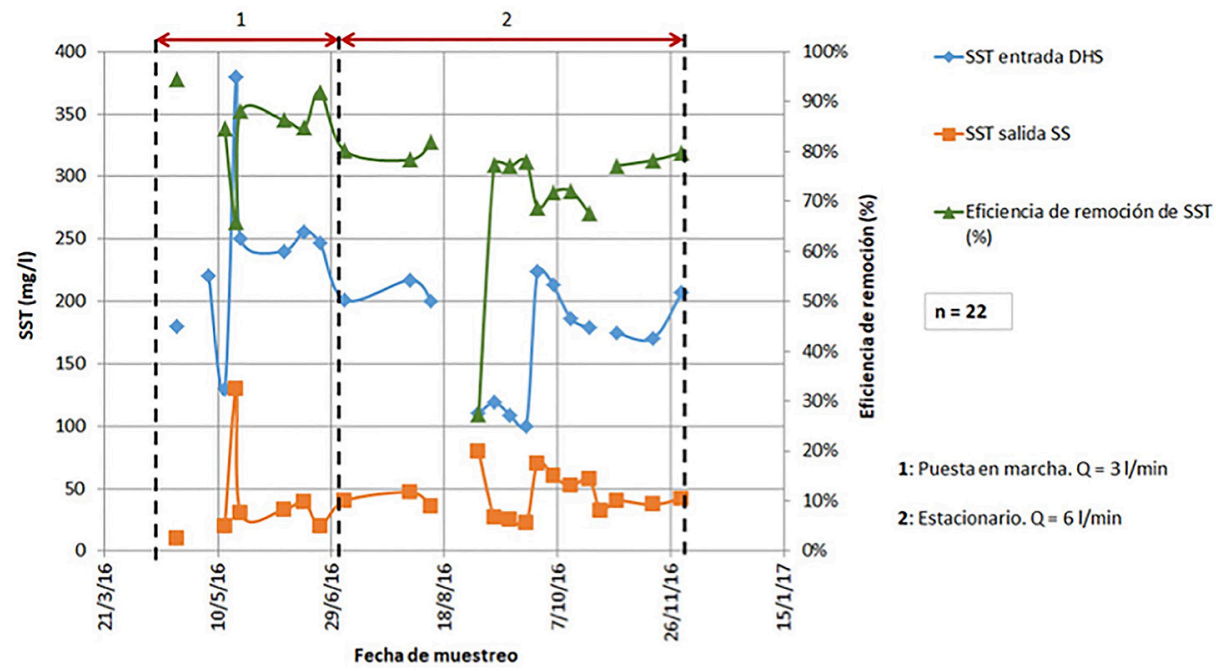

Figura 7. Variación de los SST en el sistema de DHS durante el período de evaluación

El comportamiento con respecto a este parámetro fue menos favorable al compararlo con el de los parámetros de DBO y DQO. Como en el caso anterior, se tuvo un valor muy elevado de este parámetro durante la puesta en marcha, sin embargo el problema también se presentó durante el mes de octubre del 2016, período en el que se trabajó a una carga de 6 1/min y se sobrepasó ligeramente el límite de vertido vigente de $50 \mathrm{mg} / \mathrm{l}$. 
El problema se pudo deber a un arrastre de lodos, que no fueron retenidos en el sedimentador secundario; pues, como se verá más adelante, algunos problemas de arrastre de sólidos se presentaron durante la operación del sistema.

En la siguiente tabla se muestra una síntesis de los valores para los tres parámetros analizados durante el período de evaluación.

En general, los resultados obtenidos muestran que el reactor DHS presenta una calidad del agua aceptable para los estándares de Costa Rica (DE-33601-S-Minae, 2007) una vez que se estabiliza y opera a cargas aceptables para la tecnología. Este punto se retomará más adelante.

Adicionalmente, en la siguiente figura se presentan algunas fotografías del efluente para las fechas y condiciones operativas especificadas.

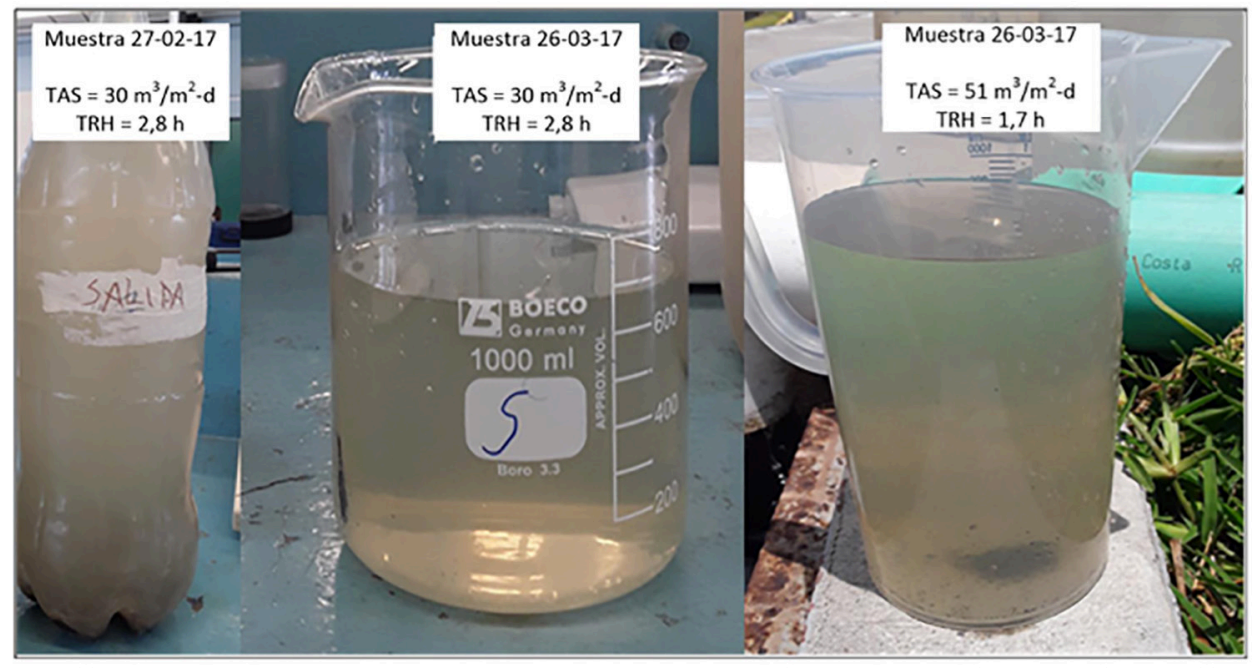

Figura 8. Aspecto visual del efluente final de DHS para distintos momentos de la operación

Las tres fotografías corresponden al período 4 de recuperación del sistema, siendo la primera correspondiente al inicio de la recuperación (arrastre de biomasa que fue lavada de la espuma), la segunda foto corresponde al momento de estabilización del efluente (efluente de alta calidad visual y con ausencia de olores) y la tercera fotografía corresponde al aspecto del efluente final después de algunas semanas de operar a una TAS de $51 \mathrm{~m}^{3} / \mathrm{m}^{2}$-d, equivalente a un TRH de únicamente $1,7 \mathrm{~h}$. Se observa que hubo un daño importante de la calidad visual del efluente con respecto al de la segunda fotografía, con un arrastre de sólidos (problemas de capacidad de sedimentador secundario) y con una turbidez muy elevada en el efluente.

Por último, en la siguiente tabla se presentan los resultados obtenidos para los análisis realizados por el Laboratorio Nacional de Aguas AyA para la entrada y salida al sistema DHS + SS en junio y julio del 2017. 
Los resultados del Laboratorio Nacional de Aguas AyA concuerdan con los reportados para las fechas de esos muestreos, cuando el sistema dio excelentes resultados a nivel de calidad del agua tratada en pleno cumplimiento de la norma de vertido vigente.

Como punto adicional interesante, en ambos análisis se observa una disminución del nitrógeno amoniacal en el sistema para producir un aumento en el nitrato. Se evidencia por lo tanto una nitrificación parcial del agua residual. Este tema debería estudiarse con mayor detalle en futuras investigaciones. Aun cuando no es un contaminante normado en Costa Rica, el nitrógeno puede afectar los cuerpos de agua superficial y esta tecnología podría ser una solución para la reducción de este elemento por medio de los procesos biológicos de nitrificación/desnitrificación.

Tabla 2. Síntesis de resultados de análisis de calidad de agua de entrada y salida de reactor DHS.

\begin{tabular}{lccccccccc}
\hline & \multicolumn{3}{c}{ DBO (mg/l) } & \multicolumn{2}{c}{ DQO (mg/l) } & & \multicolumn{2}{c}{ SST (mg/l) } \\
& Entrada & Salida & $\begin{array}{c}\text { Efiencia } \\
\text { de } \\
\text { remoción }\end{array}$ & Entrada & Salida & $\begin{array}{c}\text { Eficiencia } \\
\text { de } \\
\text { remoción }\end{array}$ & Entrada & Salida & $\begin{array}{c}\text { Eficiencia } \\
\text { de } \\
\text { remoción }\end{array}$ \\
\hline $\begin{array}{l}\text { Número de } \\
\text { Mediciones }\end{array}$ & 28 & 30 & - & 29 & 29 & - & 22 & 22 & - \\
$\begin{array}{l}\text { Promedio } \\
\text { Valor }\end{array}$ & 92 & 35 & $59 \%$ & 219 & 83 & $61 \%$ & 196 & 43 & $77 \%$ \\
$\begin{array}{l}\text { Máximo } \\
\text { Valor }\end{array}$ & 214 & 68 & $85 \%$ & 369 & 209 & $81 \%$ & 380 & 130 & $94 \%$ \\
$\begin{array}{l}\text { Mínimo } \\
\begin{array}{l}\text { Desviación } \\
\text { Estándar }\end{array}\end{array}$ & 62 & 23 & $24 \%$ & 131 & 45 & $42 \%$ & 100 & 10 & $27 \%$ \\
\hline
\end{tabular}

Tabla 3 Resultados de Laboratorio Nacional de Aguas AyA

\begin{tabular}{|c|c|c|c|c|c|}
\hline \multirow[b]{2}{*}{ Parámetro } & \multirow[b]{2}{*}{ Unidades de medición } & \multicolumn{2}{|c|}{ Muestreo 01-06-2016 } & \multicolumn{2}{|c|}{ Muestreo 06-07-2016 } \\
\hline & & Entrada DHS & Salida SS & Entrada DHS & Salida SS \\
\hline Oxígeno Disuelto & $\mathrm{mg} / 1$ & 4 & 5,5 & 2,8 & 2,8 \\
\hline DBO & $\mathrm{mg} / 1$ & 89 & 22 & 74 & 35 \\
\hline DQO & $\mathrm{mg} / 1$ & 119 & 46 & 208 & 61 \\
\hline $\begin{array}{l}\text { Sólidos } \\
\text { Suspendidos } \\
\text { Totales }\end{array}$ & $\mathrm{mg} / 1$ & 36 & 16 & 64 & 32 \\
\hline $\begin{array}{l}\text { Sólidos } \\
\text { Sedimentables }\end{array}$ & $\mathrm{ml} / 1$ & 0,5 & 0 & 0 & 0 \\
\hline $\begin{array}{l}\text { Nitrógeno } \\
\text { amoniacal }\end{array}$ & $\mathrm{mg} / 1$ & 22 & 7,8 & 56 & 25 \\
\hline Nitrato & $\mathrm{mg} / 1$ & 2,7 & 52 & 6,8 & 28 \\
\hline Fósforo & $\mathrm{mg} / 1$ & 1,7 & 0,3 & 3 & 0,4 \\
\hline
\end{tabular}




\subsection{Operación y Mantenimiento del sistema}

Durante el período de evaluación, además de obtener valores de los principales parámetros de calidad del agua tratada, se dio seguimiento a las condiciones de operación del sistema. Los resultados más significativos de esta experiencia se presentan a continuación.

Debido a la condición de trabajar en un planta piloto, el caudal a enviar al sistema era muy bajo (menor a 12 1/min ó $17 \mathrm{~m}^{3} / \mathrm{d}$ ). Regular este caudal por medio de una válvula de compuerta fue complicado debido al problema de colmatación de esa unidad al trabajar prácticamente cerrada. Esto produjo que en ocasiones el sistema quedara sin alimentación por algunas horas. Este choque de cargas produjo problemas en el reactor, particularmente en una ocasión en la que el sistema pasó al menos un día sin alimentación. El resultado fue que la biomasa muriera y saliera del sistema al reactivar el flujo. En la siguiente figura se muestran algunas fotografías del estado del sedimentador secundario tras el desprendimiento de la biomasa.

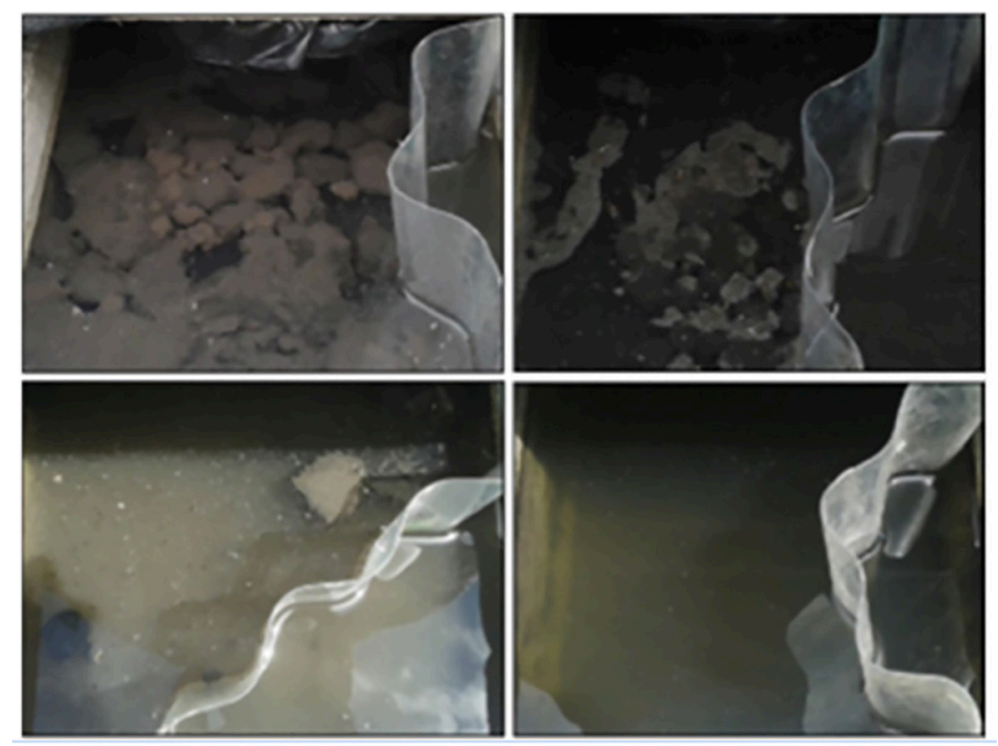

Figura 9. Desprendimiento de biomasa debido a problemas operativos en el reactor DHS. Fuente: Rodríguez, 2017

El valor tan elevado de SST durante el mes de octubre podría deberse a este fenómeno. El sedimentador secundario de limpieza manual fue incapaz de retener este exceso de sólidos y el efluente los arrastró, afectando la calidad de los análisis. Este problema no debería presentarse normalmente en una PTAR a escala completa debido a la continuidad en la alimentación del caudal. El reactor logró estabilizarse aproximadamente una semana después de volver a tener flujo.

Otro problema operativo que se observó durante la experiencia fue el de aparición de vectores como polillas y gusanos. En la siguiente figura se muestran algunas fotografías de este problema. 
En el caso de las "moscas de agua" o "palomillas", se formaron en la parte superior del reactor. Este problema es usual en los filtros percoladores, y las fuentes bibliográficas especializada (Metcalf \& Eddy, 2014; Water Environment Federation, 2010) recomiendan un sistema de distribución del agua más eficiente para garantizar que toda la superficie se moje de manera cíclica. De este modo se elimina la posibilidad de que estos insectos tengan espacios secos para desarrollarse. En una planta de escala completa se debería pensar en un sistema móvil que permita un mojado homogéneo sobre toda la superficie.

Con respecto a los gusanos, en condiciones de operación adecuados a nivel de calidad del agua en el efluente se encontraron cantidades importantes de larvas en una caja de registro en la que se acumulaba el agua tratada antes de ser enviada al sistema de vertidos y escurridos de la PTAR Los Tajos. No se pudo verificar el tipo de insecto y se desconoce el origen o razón de formación del mismo. Tampoco se sabe si la procreación de este gusano puede tener efectos nocivos sobre los ecosistemas acuáticos. Se trata de un tema al que se le deberá dar seguimiento en el futuro.

Finalmente, como medida operativa adicional, debe mencionarse que hubo problemas con el sedimentador secundario de limpieza manual. El proceso de limpieza no siempre se realizaba y sucedía que la unidad se llenaba con lodos biológicos que eran finalmente arrastrados con el efluente final. En el caso de una PTAR a escala real el diseño adecuado del sedimentador secundario será clave para el buen funcionamiento del sistema.

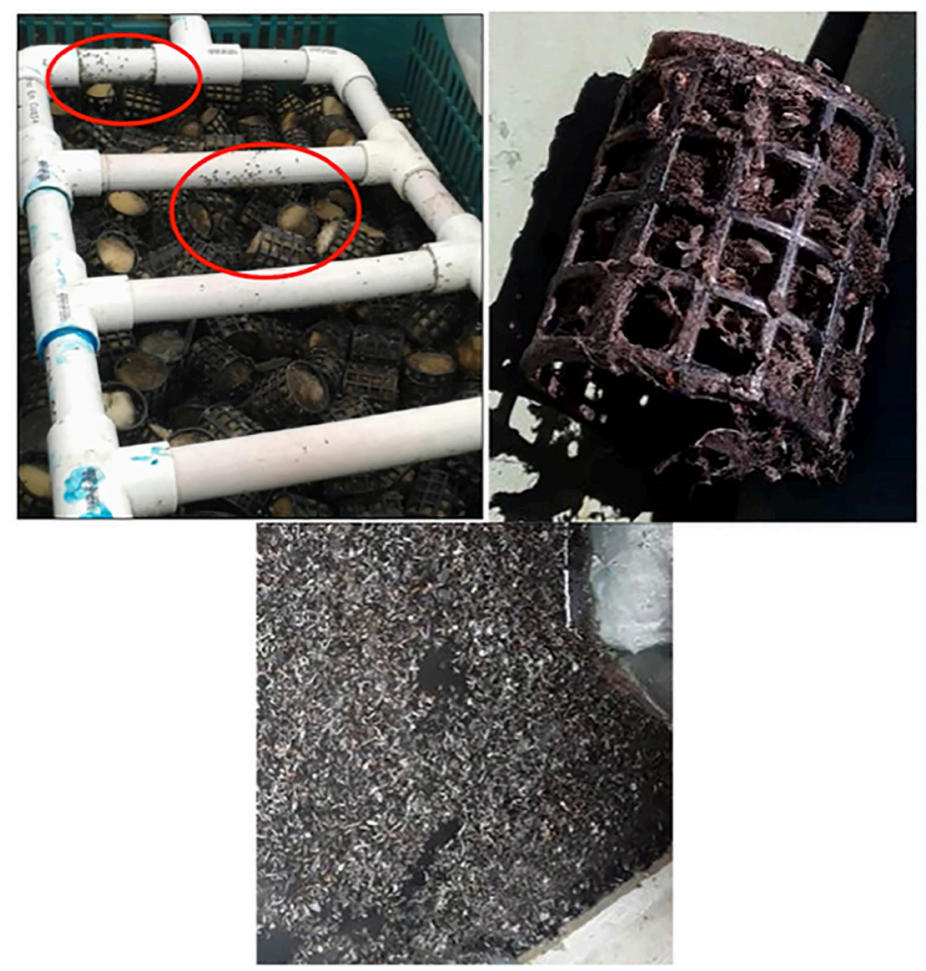

Figura 10. Generación de vectores en reactor DHS 


\section{ANÁLISIS DE RESULTADOS}

En esta sección se interpretan los resultados más importantes que se obtuvieron durante el funcionamiento de la planta piloto. Se hace énfasis en las cargas de operación del sistema, la calidad del efluente que se puede obtener con esta tecnología y algunas recomendaciones a nivel de diseño que deberían plantearse para el éxito de la tecnología en una PTAR a escala real.

\subsection{Cargas del sistema DHS}

Para caracterizar las cargas a las que se impuso el sistema DHS se usaron dos indicadores: la carga orgánica volumétrica y la tasa de aplicación hidráulica superficial. La definición matemática de ambos indicadores se presenta a continuación:

$$
\operatorname{COV}=\frac{Q \cdot D B O_{\text {aplicada }}}{V_{D H S}}
$$

En donde

COV: carga orgánica volumétrica $\left(\mathrm{kgDBO} / \mathrm{m}^{3}-\mathrm{d}\right)$

Q: $\quad$ caudal afluente en el reactor DHS $\left(\mathrm{m}^{3} / \mathrm{d}\right)$

$\mathrm{DBO}_{\text {aplicada }}$ : concentración en DBO del agua residual de entrada en el reactor DHS $\left(\mathrm{kg} / \mathrm{m}^{3}\right)$ $\mathrm{V}_{\mathrm{DHS}}$ : volumen de esponja del reactor DHS $\left(\mathrm{lm}^{3}\right)$

$T A H=\frac{Q}{A_{D H S}}$

En donde

TAH: tasa de aplicación hidráulica superficial $\left(\mathrm{m}^{3} / \mathrm{m}^{2}-\mathrm{d}\right)$

Q: $\quad$ caudal afluente al reactor DHS $\left(\mathrm{m}^{3} / \mathrm{d}\right)$

ADHS: área superficial del reactor DHS $\left(0,28 \mathrm{~m}^{2}\right)$

En la siguiente figura se muestra la evolución de la COV y de la TAH durante el período de evaluación.

Para el caso de la COV, el valor reportado varió entre 0,31 a 1,85 kgDBO $/ \mathrm{m}^{3}-\mathrm{d}$, siendo el promedio de la carga aplicada de $0,72 \mathrm{kgDBO} / \mathrm{m}^{3}$-d para el período de evaluación. Para la TAH el valor varió linealmente con el caudal de entrada, pasando desde 15,3 hasta $51,4 \mathrm{~m}^{3}-\mathrm{m}^{-2}-\mathrm{d}^{-1}$, siendo el valor de $30,8 \mathrm{~m}^{3}-\mathrm{m}^{-2}-\mathrm{d}^{-1}$ el más usado durante el experimento (correspondiente al caudal de $61 / \mathrm{min}$ ). 


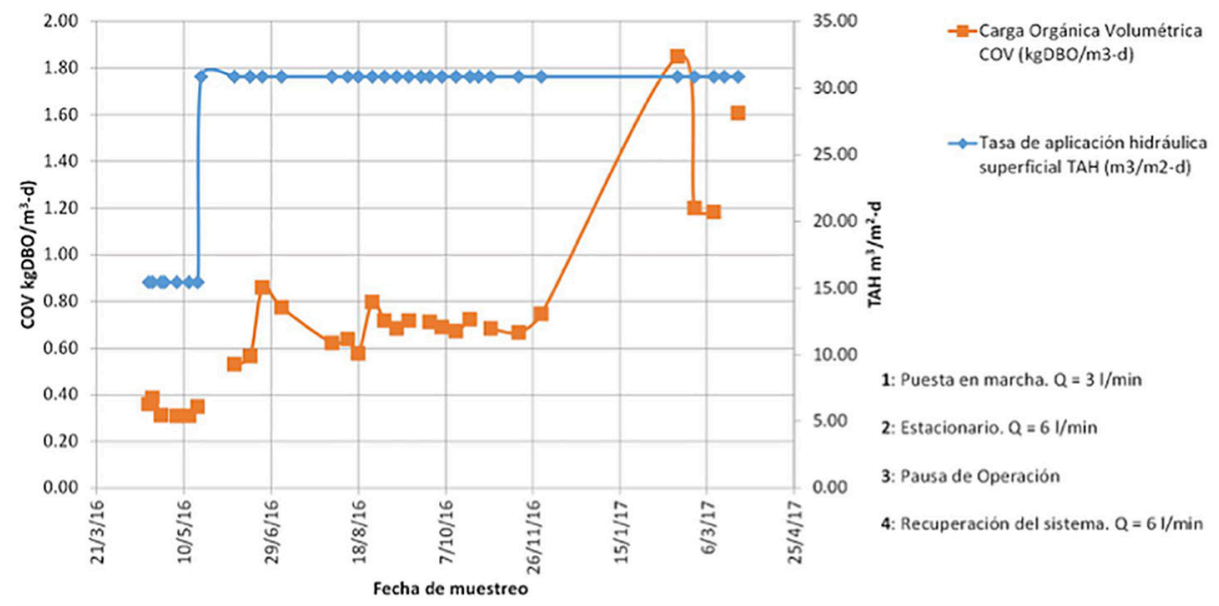

Figura 11. Cargas de operación del reactor DHS.

Para el caso de filtros percoladores como postratamiento de sedimentadores primarios Metcalf \& Eddy (2014) propone un rango para la remoción de DBO de entre 0,6 a $2,4 \mathrm{kgDBO} / \mathrm{m}^{3}$-d cuando se usa medio de soporte plástico y para obtener porcentajes de remoción de DBO de entre 70 a $90 \%$ en el sistema. Para el período de funcionamiento estacionario (fase 2 y 4 ) se mantuvo una COV muy cercana a ese rango $\left(0,53\right.$ a $\left.1,85 \mathrm{kgDBO} / \mathrm{m}^{3}-\mathrm{d}\right)$ con resultados favorables sobre la calidad del efluente. Para el caso del TAS, en el caso del material plástico, las fuentes bibliográficas (Metcalf \& Eddy, 2014) reportan valores de entre 10 hasta $75 \mathrm{~m}^{3}-\mathrm{m}^{-2}-\mathrm{d}^{-1}$ para los filtros percoladores de alta tasa. En ambos casos, el relleno del filtro con esponjas de poliuretano opera adecuadamente dentro de la primera mitad de esos rangos de carga, según los resultados obtenidos en esta investigación.

\subsection{Calidad del agua tratada y confiabilidad del sistema}

En la siguiente figura se observa el comportamiento de los datos experimentales obtenidos únicamente para las condiciones de TAH de 30,8 $\mathrm{m}^{3}-\mathrm{m}^{-2}-\mathrm{d}^{-1}$ (TRH de 2,8 h), valor operativo considerado como el más apropiado para la operación del sistema según lo expuesto anteriormente. En la figura se puede percibir la dispersión de los datos para los parámetros de entrada y salida de la DBO, la DQO y los SST para ese período.

Al examinar el gráfico de cajas se puede observar que, para los tres parámetros evaluados, la dispersión de los valores de concentración a la salida del reactor DHS es siempre menor que la dispersión a la entrada. Esto significa que, ante picos a la entrada del sistema, el reactor tendría capacidad de amortiguarlos en alguna medida y seguir entregando un efluente de calidad que cumpla con la reglamentación vigente.

Luego, para los parámetros de DBO y DQO el sistema cumplió con límites de vertido (señalados con una línea roja de mayor espesor) en todos los casos. Para los SST, el límite máximo permitido en la reglamentación nacional corresponde 
aproximadamente al tercer cuartil; es decir, aproximadamente el 25\% de los valores obtenidos en el efluente superaron el límite de $50 \mathrm{mg} / \mathrm{l}$ para la condición operacional más favorable.

Esto indica que se tuvo problemas con el cumplimiento del límite de SST. Se presume que este problema estuvo relacionado con el funcionamiento del sedimentador secundario más que con el reactor DHS. La función de esta unidad es la de retener la biomasa desprendida del medio de esponja. Durante la operación del sistema se observó algunos problemas de arrastre en el sedimentador secundario que también afectarían negativamente el valor del parámetro de SST en el efluente final como se mencionó anteriormente. Es necesario profundizar en este tema por medio de nuevos ensayos experimentales para validar esta hipótesis.

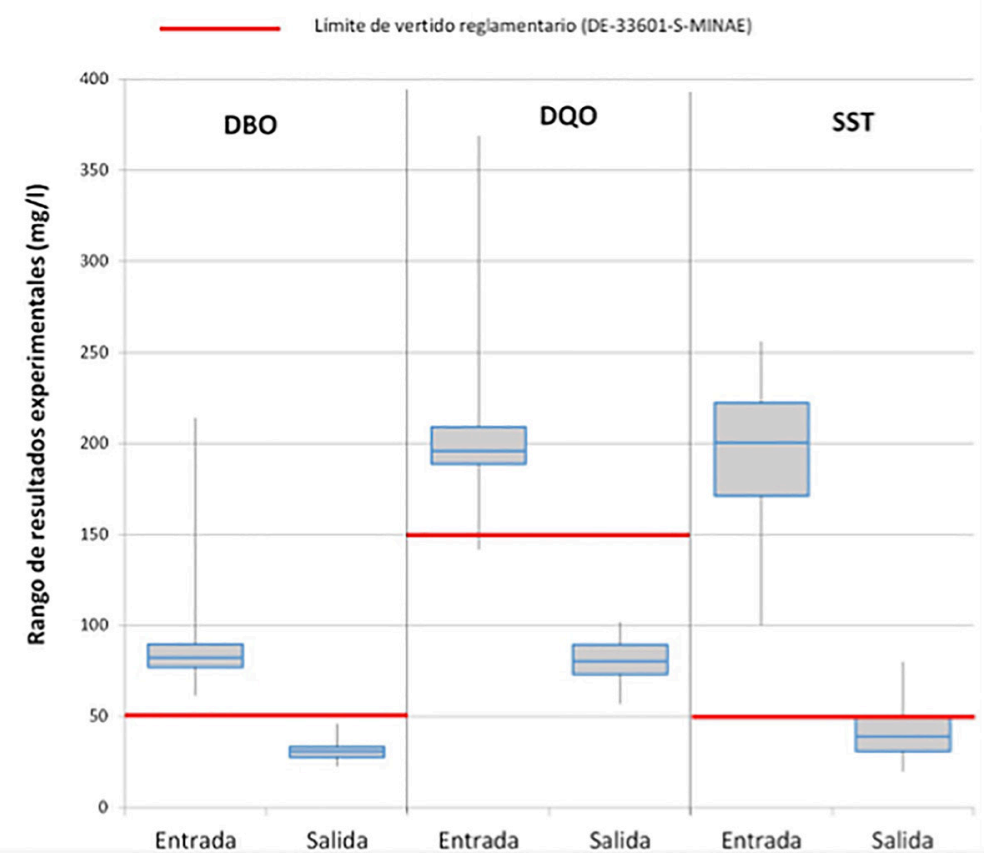

Figura 12. Comportamiento del sistema DHS durante condición estacionaria de TAH de $30,8 \mathrm{~m}^{3}-\mathrm{m}^{-2}-\mathrm{d}^{-1}$ (TRH de 2,8h)

\subsection{Perspectivas de la tecnología en Costa Rica}

Dado los resultados obtenidos con la planta piloto, es posible asegurar que la tecnología de DHS podría usarse para el cumplimiento de los parámetros de contaminación por materia orgánica (DBO, DQO y SST) de la reglamentación vigente (DE-33601S-Minae, 2007) para el caso de las aguas residuales ordinarias. Si se considera una TAH de un máximo de $30,8 \mathrm{~m}^{3}-\mathrm{m}^{-2}-\mathrm{d}^{-1}$ (TRH de $2,8 \mathrm{~h}$ y COV cercana a $0,70 \mathrm{kgDBO} /$ $\mathrm{m}^{3}-\mathrm{d}$ ), y bajo condiciones operativas adecuadas, el sistema debería cumplir con los límites máximos establecidos en ese reglamento. 
Dos posibles aplicaciones prácticas a nivel de operación unitaria de esta tecnología, compatibles con la planta piloto monitoreada podrían ser:

- Reactor DHS como postratamiento de un sedimentador primario convencional, tal y como se probó en este caso. La primera unidad removería parte de los SST que podrían obstruir el sistema. Los lodos primarios y secundarios generados en el proceso podrían mezclarse para su tratamiento común (Water Environment Federation, 2010).

- Reactor DHS como postratamiento de un sedimentador digestor, tanque séptico, fosa Imhoff o similar. La primera unidad de tratamiento primario tendría por función la remoción de los SST, igual que en el caso de un tratamiento primario (con una eficiencia equivalente). Sin embargo, este proceso tiene la ventaja de que los lodos primarios no requieren un tratamiento inmediato, pues la unidad se diseña para almacenar y estabilizar los sólidos por vía anaerobia. Los lodos secundarios recuperados en el sedimentador secundario podrían enviarse a digestión en el proceso de tratamiento primario. Este proceso podría ser interesante para pequeñas comunidades con limitaciones de espacio, en donde se colocaría un reactor DHS en lugar de un humedal construido o "biojardinera", por ejemplo. Su eficacia debería probarse a nivel de planta a escala real.

El reactor DHS se ha estudiado ampliamente para el postratamiento de efluentes anaerobios de reactores UASB (Bressani Ribeiro, 2015; Harada, 2015; Sato, Okubo, Onodera, Ohashi, \& Harada, 2006; Tandukar et al., 2005) resultando en efluentes de calidad suficiente para su vertido a cuerpos de agua superficial según la reglamentación de Costa Rica. Esta es también una posibilidad que no ha sido analizada en nuestro país. El reactor UASB brinda un efluente de una calidad superior al de un sedimentador primario y también puede operar como un digestor de los lodos secundarios.

Luego, con respecto a las ventajas de la tecnología de DHS se puede citar lo siguiente:

- El tiempo de retención hidráulica (TRH) de solo 2.8 horas garantiza un volumen reducido para el reactor. Suponiendo un porcentaje de espuma en el volumen total del reactor de aproximadamente $40 \%$, el TRH total del reactor sería de aproximadamente 7 horas. A modo de comparación, el TRH de un sistema de lodos activados convencional ronda las 8 a 12 horas (Von Sperling, 2014). Esto representaría un reactor DHS con aproximadamente un 60 a $90 \%$ el volumen útil de un reactor aerobio, con los ahorros respectivos de obra civil y de espacio.

- A nivel energético, el sistema sería significativamente menos demandante que otras tecnologías aerobias como los lodos activados. Esto se debe a que en el DHS se requeriría únicamente de un brazo de distribución del agua de entrada y de un ventilador en el fondo para el suministro del aire. Ambos equipos podrían omitirse si se diseña un sistema de distribución hidráulico ( ventilación natural (sin ventilador) es suficiente para suministrar el oxígeno requerido durante la oxidación biológica. A modo de referencia, Sperling (Von Sperling, 2005) sugiere que para sistemas de lodos activados convencionales el consumo energético ronda los 18 a 26 kWh-habitante-año para el proceso biológico. Los sistemas de tratamiento primario y filtro percolador podrían tener incluso un consumo energético nulo en caso de optar por una distribución hidráulica y ventilación natural de la tecnología. Este aspecto debería evaluarse para cada caso específico. 
Algunas desventajas de la tecnología DHS son:

- En el caso de Costa Rica, no se cuenta con un proveedor local del material de relleno del filtro percolador (espuma de poliuretano), por lo que de momento tendría que importarse del extranjero. El costo aproximado de este relleno es de unos 1000 dólares el metro cúbico cuando se importa, condición que debería tomarse en cuenta durante la fase de análisis de opciones tecnológicas. Trejos (2017) demostró que este costo podría reducirse sustancialmente si el material se produce de manera local.

- No existe experiencia en sistemas de escala real para esta tecnología en Costa Rica. Antes de generalizarse su uso (como para cualquier tecnología) se debe continuar la investigación en el proceso a una escala real (y no en un reactor piloto) así como dar capacitación a la comunidad técnica nacional sobre el funcionamiento de la tecnología y sobre las necesidades operativas de la misma.

- En el caso de continuar con la investigación a escala de planta real para esta tecnología deberá considerarse realizar las siguientes mejoras con respecto a la planta piloto:

- Usar como postratamiento de sedimentador primario o similar.

- Puede considerarse una TAH de 30,8 $\mathrm{m}^{3}-\mathrm{m}^{-2}-\mathrm{d}^{-1}$ (TRH de 2,8h y una COV de entre 0,60 a $0,80 \mathrm{kgDBO} / \mathrm{m}^{3}-\mathrm{d}$ ) como adecuada para el diseño de la unidad.

- Debe mejorarse el sistema de distribución del agua cruda, colocando un sistema mecanizado que permita un mojado homogéneo de toda la superficie con el fin de evitar la formación de espacios de desarrollo para larvas y mosquitos y para reducir los volúmenes muertos del reactor DHS.

- El sedimentador secundario deberá diseñarse y construirse apropiadamente, considerando las condiciones de carga hidráulica superficial, profundidad y método de remoción de lodos necesarias para su adecuado funcionamiento. Podría eventualmente considerarse la opción de recirculación del efluente final de esta unidad hacia el reactor DHS, como se hace usualmente para los sistemas de filtro percolador de alta tasa.

- La aireación del reactor DHS es otro aspecto que debe revisarse con cuidado, verificando que el gradiente de temperatura podría ser suficiente para producir el flujo de aire necesario, o por el contrario, suministrando un sistema de ventilación de fondo para esta función.

\section{CONCLUSIONES}

El seguimiento de una planta piloto de un reactor de esponjas colgantes de flujo descendente (DHS por sus siglas en inglés) demostró que la tecnología es capaz de cumplir para los parámetros de DBO, DQO y SST (para este último parámetro con algunas reservas) en el caso de ser usado como postratamiento de un Sedimentador Primario, cuando opera a una carga orgánica volumétrica (COV) de entre 0,60 a 0,80 $\mathrm{kgDBO} / \mathrm{m} 3$-d y una Tasa de aplicación hidráulica superficial (TAH) cercana a $30 \mathrm{~m} 3$ / $\mathrm{m} 2$-d (TRH de 2,8 horas con respecto al volumen de esponja). Una vez estabilizado el reactor DHS la calidad del efluente mostró poca dispersión, aun cuando se registraron picos de carga orgánica a la entrada del sistema. 
La tecnología DHS resulta entonces una opción interesante para el tratamiento de las aguas residuales ordinarias en Costa Rica, particularmente cuando se compara con tecnologías de mayor demanda de energía y espacio como los lodos activados. Con esta investigación se valida la tecnología como apta para las condiciones de Costa Rica, validando las investigaciones de otros países realizadas en el tema.

Se recomienda ampliar la escala de prueba de la tecnología y usar el sistema de DHS en una planta de tratamiento a escala plena. Para un mejor desempeño del sistema deberá diseñarse un sistema de distribución del agua de alimentación y un sedimentador secundario adecuados.

La tropicalización de esta tecnología deberá ir acompañada de una capacitación en el funcionamiento y operación del sistema para la comunidad técnica nacional.

\section{AGRADECIMIENTOS}

Se agradece a la agencia de cooperación japonesa, a la empresa NJS y a la Universidad Tecnológica de Nagaoka por el acompañamiento y donación del material de soporte DHS para realizar estas pruebas. Adicionalmente, se agradece a la administración de la PTAR Los Tajos por permitir instalar el reactor piloto dentro de sus instalaciones y darle el mantenimiento necesario, y al laboratorio Ambiental de la Escuela de Ingeniería Civil de la Universidad de Costa Rica por poner a disposición sus equipos para la realización de los análisis de laboratorio.

\section{REFERENCIAS}

AYA, MINAE, \& MINSA. Política Nacional de Saneamiento en Aguas Residuales (2016). San José.

Bressani Ribeiro, T. (2015). Sistema UASB/FBP submetido a hidrograma típico de vazão: avaliação do uso de espuma de poliuretano e operação sem decantadores secundários. Tesis de Maestría, Universidad Federal de Minas Gerais, 1-173.

DE-33601-S-Minae. (2007). Reglamento de vertido y reuso de aguas residuales, (55), 56.

Harada, H. (2015). Introducción a la Tecnología Sostenible del Tratamiento de Aguas Residuales mediante el Reactor Anaeróbico de Manto de Lodo de Flujo Ascendente (UASB) y el Reactor DHS (Down-flow Hanging Sponge). Curso Internacional En Japón.

Mahmoud, M., Tawfik, A., \& El-Gohary, F. (2011). Use of down-flow hanging sponge (DHS) reactor as a promising post-treatment system for municipal wastewater. Chemical Engineering Journal, 168(2), 535-543. https://doi.org/10.1016/j.cej.2011.01.019

Metcalf \& Eddy. (2014). Wastewater engineering : treatment and resource recovery. (McGrawHill, Ed.) (4th ed.).

Murillo, A. (2017). Análisis comparativo de tecnologías para el tratamiento colectivo de las aguas residuales en poblaciones menores a 5000 personas equivalentes en Costa Rica. Tesis de Licenciatura, Universidad de Costa Rica. 
Rodríguez, D. (2017). Monitoreo y evaluación de una planta piloto para el tratamiento de agua residual por medio de un sistema de biomasa adherida con esponjas colgantes de flujo descendente, implementada como tratamiento secundario para el efluente de un sedimentador primario. Tesis de Licenciatura, Universidad de Costa Rica.

Sato, N., Okubo, T., Onodera, T., Ohashi, A., \& Harada, H. (2006). Prospects for a selfsustainable sewage treatment system: A case study on full-scale UASB system in India's Yamuna River Basin. Journal of Environmental Management, 80(3), 198-207. https://doi.org/10.1016/j.jenvman.2005.08.025

Tandukar, M., Uemura, S., Machdar, I., Ohashi, A., \& Harada, H. (2005). A low-cost municipal sewage treatment system with a combination of UASB and the "fourthgeneration" downflow hanging sponge reactors. Water Science and Technology, 52(1-2), 323-329.

Tawfik, A., Ohashi, A., \& Harada, H. (2006). Sewage treatment in a combined up-flow anaerobic sludge blanket (UASB)-down-flow hanging sponge (DHS) system. Biochemical Engineering Journal. https://doi.org/10.1016/j.bej.2005.11.018

Trejos, D. (2017). Proyecto de investigación de materiales para implementación de sistema de tratamiento DHS. Universidad de Costa Rica.

Uemura, S., Okubo, T., Maeno, K., Takahashi, M., Kubota, K., \& Harada, H. (2016). Evaluation of water distribution and oxygen mass transfer in sponge support media for a down-flow hanging sponge reactor. International Journal of Environmental Research, 10(2), 265-272.

Uemura, S., Suzuki, S., Maruyama, Y., \& Harada, H. (2012). Direct treatment of settled sewage by DHS reactors with different sizes of sponge support media. International Journal of Environmental Research, 6(1), 25-32.

Von Sperling, M. (2005). Introdução à qualidade das águas e ao tratamento de esgotos. (UFMG, Ed.), Princípios do tratamento biológico de águas residuárias. Belo Horizonte.

Von Sperling, M. (2007). VOL_5_Activated Sludge and Aerobic Biofilm Reactors. (UFMG, Ed.), Biological Wastewater Treatment series. Belo Horizonte.

Von Sperling, M. (2014). Lodos Ativados. Princípios do tratamento biológico de águas residuárias. (UFMG, Ed.) (4th ed.). Belo Horizonte.

Water Environment Federation. (2010). Design of Municipal Wastewater Treatment Plants. WEF Manual of Practice No. 8, 3034. Retrieved from http://cedb.asce.org/ cgi/WWWdisplay.cgi?9203843 